\title{
In silico evidence supports a role for DNA methylation in Sirt1-mediated effects of dietary restriction
}

\author{
L. J. Ions, D. Swan and D. Ford \\ Institute for Cell and Molecular Biosciences, Newcastle University, Newcastle upon Tyne, NE2 4HH, UK
}

Dietary restriction (DR) remains one of the most robust dietary interventions proved effective to increase lifespan across evolutionarily distinct species, from yeast to rodents ${ }^{(1)}$. The NAD-dependent (class III) histone deacetylase Sirt1 in mammals, and its orthologue in other species, may be pivotal in mediating the effect of DR to increase lifespan. Ageing is associated with changes in genome methylation (e.g. ${ }^{(2)}$, which may be causative in the ageing process. Such observations, in view of the activity of Sirt1 at the level of the epigenome, form the basis for our hypothesis that epigenetic effects of Sirt1 activity mediate some of the beneficial effects of DR that contribute to lifespan extension.

Our approach to testing this hypothesis was to determine the overlap between genes that bind Sirt1, those regulated at the mRNA level in response to DR and those that show an age-related change in methylation status, predicted on the principle that overlaps greater than would be expected by chance would support our hypothesis.

By representing all genes using ENSEMBL identifiers, we identified overlaps between a list of 418 genes identified as interacting physically with the histone deacetylase $\operatorname{Sirt}^{(3)}$, a list of 2954 genes found to respond to DR, compiled from 11 published studies, and a list of 1040 genes whose DNA methylation status changed in mouse small intestine in association with ageing ${ }^{(2)}$. All overlaps were found to be greater than would be expected by chance using the principle of hypergeometric distribution, as shown in Table 1. The promoter regions of 80 of the 91 genes that responded to DR and also bound Sirt1 (i.e. 88\%) were found to include CpG islands (defined as having a GC content of greater that $50 \%$ and $\mathrm{CpG}$ observed/expected ratio of greater than $0.6^{(5)}$ ), representing sites of potential differential methylation in ageing tissues; this proportion is also greater than would be expected by chance (representation factor $=1.47$, $\left.P=3.395 \times 10^{-9}\right)$, based on the assumption that $60 \%$ of mouse genes have associated CpG islands.

Table 1. Overlaps between genes responsive to DR, genes that bind Sirt1 and genes that show changes in methylation status with ageing, based on in silico analysis. ' $n$ ' indicates the number of genes common to both groups; RF indicates the representation factor (i.e. observed/expected); $P$ indicates the hypergeometric probability

\begin{tabular}{lcc}
\hline & Genes binding Sirt1 (418 genes) & $\begin{array}{c}\text { Genes that show methylation } \\
\text { changes with ageing (1040 genes) }\end{array}$ \\
\hline Genes responsive to DR (2954) & $n 91 ; \mathrm{RF}=1.60 ; P=2.695 \times 10^{-6}$ & $n 191 ; \mathrm{RF}=1.27 ; P=5.47 \times 10^{-5}$ \\
Genes binding Sirt1 (418 genes) & $n 37 ; \mathrm{RF}=2.05 ; P=1.66 \times 10^{-5}$ \\
\hline
\end{tabular}

Overall, this in silico analysis supports our hypothesis that Sirt1-mediated effects of DR include effects on DNA methylation. Future studies should examine directly the extent to which DR and/or Sirt1 expression influence genome methylation status and should determine if such effects are causal with respect to ageing and/or lifespan extension.

Funded by a BBSRC studentship to LJI.

1. Guarente L \& Picard F (2005) Calorie restriction - the SIR2 connection. Cell 120, 473-482.

2. Maegawa S, Hinkal G, Kim HS et al. (2010) Widespread and tissue specific age-related DNA methylation changes in mice. Genome Res (Epublication ahead of print version).

3. Oberdoerffer P, Michan S, McVay M et al. (2008) SIRT1 redistribution on chromatin promotes genomic stability but alters gene expression during aging. Cell 135, 907-918.

4. Gardiner-Garden M \& Frommer M (1987) CpG islands in vertebrate genomes. J Mol Biol 196, 261-282.

5. Wang Y \& Leung FCC (2004) An evaluation of new criteria for $\mathrm{CpG}$ islands in the human genome as gene markers. Bioinformatics 20, 1170-1177. 